\title{
Adalimumab effectively reduces the rate of anterior uveitis flares in patients with active ankylosing spondylitis: results of a prospective open-label study
}

\author{
M Rudwaleit, ${ }^{1}$ E Rødevand, ${ }^{2}$ P Holck, ${ }^{3}$ J Vanhoof, ${ }^{4}$ M Kron, ${ }^{5}$ S Kary, ${ }^{5}$ H Kupper ${ }^{5}$
}

${ }^{1}$ Charité University Medicine Berlin, Benjamin Franklin Campus, Berlin, Germany; ${ }^{2} \mathrm{St}$ Olavs Hospital, Trondheim, Norway: ${ }^{3}$ Regionshospitalet Silkeborg, Silkeborg, Denmark; ${ }^{4}$ University Hasselt, Hasselt, Belgium; ${ }^{5}$ Abbott $\mathrm{GmbH} \&$ Co $\mathrm{KG}$, Ludwigshafen, Germany

Correspondence to: M Rudwaleit, Charité, Campus Benjamin Franklin Hospital Medical Department I,

Rheumatology,

Hindenburgdamm 30, 12200

Berlin, Germany; martin. rudwaleit@charite.de

Accepted 13 July 2008 Published Online First 28 July 2008

\section{UNLOCKED}

This paper is freely available online under the BMJ Journals unlocked scheme, see http:// ard.bmj.com/info/unlocked.dtl

\begin{abstract}
Objective: To evaluate the effect of adalimumab on the frequency of anterior uveitis (AU) flares in patients with active ankylosing spondylitis (AS).

Methods: We determined the history of ophthalmologistdiagnosed AU in 1250 patients with active AS who were enrolled in a multinational, open-label, uncontrolled clinical study of treatment with adalimumab, $40 \mathrm{mg}$ every other week for up to 20 weeks. All AU flares were documented throughout the adalimumab treatment period plus 70 days. We compared the rates of AU flares per 100 patient years (PYS) reported during the year before adalimumab treatment with rates during adalimumab treatment, in total and by patient subgroups.
\end{abstract}

Results: The AU flare rates before adalimumab treatment were 15/100 PYs in all patients ( $n=1250), 68.4 / 100$ PYs in 274 patients with a history of AU flares, 176.9/100 PYs in 106 patients with a recent history of AU flares, 192.9/ 100 PYs in 28 patients with symptomatic AU at baseline and 129.1/100 PYs in 43 patients with a history of chronic uveitis. During adalimumab treatment, the rate of $\mathrm{AU}$ flares was reduced by $51 \%$ in all patients, by $58 \%$ in 274 patients with a history of $\mathrm{AU}$, by $68 \%$ in 106 patients with a recent history of $\mathrm{AU}$, by $50 \%$ in 28 patients with symptomatic $\mathrm{AU}$ at baseline and by $45 \%$ in 43 patients with chronic uveitis. AU flares during adalimumab treatment were predominantly mild. Two patients with periods of high AS disease activity had new-onset AU during the treatment period.

Conclusions: Results of this prospective open-label study suggest that adalimumab had a substantial preventive effect on AU flares in patients with active AS, including patients with a recent history of AU flares.

Clinical trials: ClinicalTrials.gov Identifier: NCT00478660.

Tumour necrosis factor (TNF) antagonists are highly effective agents for the treatment of patients with active ankylosing spondylitis (AS). In addition to the spine, the immunological inflammation of AS may also involve peripheral joints and extraskeletal structures, such as the eye, skin and bowel. Between $20 \%$ and $40 \%$ of patients with AS experience at least one flare of anterior uveitis (AU) at any time during the course of the disease. ${ }^{12}$ An attack of AU may even be the first symptom that leads to the diagnosis of $\mathrm{AS}^{3}{ }^{4}$ Underlying AS is diagnosed in up to $50 \%$ of patients with $A U$, particularly in the presence of the human leukocyte antigen (HLA)-B27. ${ }^{5}$ The course of AU varies widely; patients may experience only one uveitis flare in a lifetime, whereas others have recurrent episodes. Some patients also have chronic uveitis that is characterised by persistent episodes of uveitis (defined as at least 3 months in duration) with a symptom-free interval of less than 3 months after treatment discontinuation. ${ }^{6}$

Among the traditional disease-modifying antirheumatic drugs (DMARDs) that are generally of dubious effect in patients with AS compared with patients with rheumatoid arthritis or with psoriatic arthritis, a preventive effect of DMARDs on AU flares has been reported only for sulfasalazine.' Acute or chronic episodes of AU, particularly in children with juvenile inflammatory arthritis and uveitis, have been successfully treated with infliximab, whereas etanercept was mostly ineffective. ${ }^{18-16}$ The effect of TNF antagonists on AU in patients with spondyloarthritis (SpA) or AS was analysed in one large retrospective study and one meta-analysis of seven clinical trials, four of which were placebo-controlled, randomised trials. ${ }^{17}{ }^{18}$ The retrospective study suggested that the TNF antagonists infliximab and adalimumab reduced the rate of AU flares, whereas the frequency of $\mathrm{AU}$ flares in patients with SpA who were treated with etanercept remained unchanged..$^{18}$ In the metaanalysis, infliximab and etanercept therapies reduced the incidence of AU flares, and infliximab appeared to be more effective than etanercept; adalimumab was not evaluated. ${ }^{17}$ By contrast, new-onset uveitis was reported during TNFantagonist therapy in patients with rheumatic disorders that are not commonly associated with uveitis. A review of the literature indicates that new-onset AU has been reported primarily during etanercept treatment, rarely during infliximab treatment and not during adalimumab treatment. ${ }^{11}{ }^{19-24}$ Questions of clinical interest include whether patients with $\mathrm{AS}$ and $\mathrm{AU}$ respond similarly to TNF antagonists compared with patients without a history of $A U$ and whether there is a correlation between adalimumab effectiveness on AS and on prevention of AU flares. Here, we report analyses of data from the "Review of safety and effectiveness witH Adalimumab in Patients with active ankylosing SpOnDYlitis" (RHAPSODY) trial. With 1250 patients enrolled, this is the largest prospective clinical trial to evaluate the effect of adalimumab on AU flares in patients with AS to date.

\section{PATIENTS AND METHODS}

\section{Patients}

Adults $\geqslant 18$ years of age with active AS according to the modified New York Criteria for Ankylosing Spondylitis $1984^{25}$ and with a Bath AS Disease 
Activity Index ${ }^{26}$ (BASDAI) $\geqslant 4$ despite treatment with at least one non-steroidal anti-inflammatory drug (NSAID) were enrolled. Previous treatment with another TNF antagonist was allowed if the therapy had been discontinued at least 3 weeks (for etanercept) or at least 2 months (for infliximab) before the first adalimumab injection. Ongoing treatment with NSAIDs including cyclo-oxygenase 2 inhibitors, glucocorticoids ( $\leqslant 10 \mathrm{mg} / \mathrm{dl}$ prednisolone equivalent) and/or DMARDs was allowed. The dosage regimen of current topical therapy with glucocorticoids or other agents in patients with uveitis could be tapered during the study.

\section{Study design}

This is a prespecified subanalysis of a multinational, prospective, open-label, uncontrolled clinical study of adalimumab $40 \mathrm{mg}$ every other week for the treatment of patients with AS. Patients were enrolled at 211 clinical centres in 15 European countries. This study was approved by an independent ethics committee at each centre and all patients provided written, informed consent.

Details of the study design have been published elsewhere previously. ${ }^{27}$ All patients were treated with adalimumab $40 \mathrm{mg}$ every other week for 12 weeks. Patients with documented AU flares within 1 year before and/or at baseline continued receiving adalimumab up to week 20 to provide a longer observation period. Measures of adalimumab effectiveness were collected at weeks 2, 6, 12 and 20 (as applicable). At baseline, the history of ophthalmologist-diagnosed AU was confirmed in the medical record of each patient, and the AU was assessed as acute or chronic. Chronic uveitis was defined according to The Standardization of Uveitis Nomenclature Working Group as persistent AU flares (ie, $\geqslant 3$ months in duration) with relapse occurring in the previous 3 months. ${ }^{6}$ The number of recent AU flares (in the 12 months before and/or at baseline) were documented categorically as zero, one to two and three or more episodes. Any flare of $\mathrm{AU}$ that occurred during the adalimumab treatment period and up to 70 days after the last adalimumab injection was captured as an adverse event (AE).

In addition, advanced ankylosis at baseline, defined as at least Stage IV (involvement of $\geqslant 50 \%$ of the spine in $>2$ segments), was documented by the investigators based on historical radiographs. ${ }^{28}$ The responses of spinal symptoms were evaluated by the Assessment in Spondyloarthritis International Society (ASAS) criteria as improvement by at least 20\% (ASAS20) and by at least $40 \%$ (ASAS40), ${ }^{29}{ }^{30}$ ASAS $5 / 6$ criteria (improvement of at least $20 \%$ in five of six ASAS criteria), ASAS partial remission (value of $<2$ on a $0-10$-point scale in each of the four ASAS20 domains), ${ }^{29}{ }^{30}$ and improvement by at least $50 \%$ in the BASDAI (BASDAI 50). ${ }^{26} 3132$

\section{Statistical analyses}

The methodology for this prespecified analysis of AU flare rates was described in the clinical trial protocol and statistical analysis plan before enrolment of the first patient in the study. The rate of AU flares during the entire adalimumab treatment period (including the 70-day follow-up period) was calculated as events/100 patient years (PY) for all patients enrolled in the study $(n=1250)$, and for 4 subsets of patients. Three subsets were determined by the timing of $\mathrm{AU}$ activity with respect to the baseline study visit, as follows: patients with a history of uveitis (ie, at least one AU flare anytime in the course of their $\mathrm{AS}$ ), patients with a recent AU flare (ie, at least one flare during the 12 months before and/or at baseline) and patients with symptomatic AU at baseline. The fourth subset was based on the clinical course of $\mathrm{AU}$ and included patients with chronic AU. The rate of $\mathrm{AU}$ flares during adalimumab therapy was compared with the rate of AU flares in the 12 months before baseline per 100 PYs for all patients and the four subsets of patients with a Wilcoxon signed rank test. Because the number of flares in the past 12 months had been categorically documented (ie, 0,1 to 2 , or 3 or more episodes), the total number of flares was calculated by multiplying the number of patients in the 1-2 category by 1.5 (ie, the mean of the $1-2$ category), and multiplying the number of patients in the $\geqslant 3$ category by 3 (ie, the minimum number of episodes in this category). The ASAS40 and BASDAI 50 response rates at week 12 were compared in patients with vs without a history of uveitis, and also in patients without an AU flare vs patients with at least one AU flare during adalimumab treatment.

\section{RESULTS}

\section{Clinical characteristics and AU history}

Patients with active AS ( $\mathrm{n}=1250)$ had a mean disease duration of 11 years. A history of ophthalmologist-diagnosed AU was documented in 274 of 1250 (21.9\%) enrolled patients. Of the 274 patients with AU, 43 had chronic uveitis; 106 had a recent history of uveitis and 28 had symptomatic AU at baseline.

The mean/median time of time from diagnosis of $A U$ to study enrolment was 9.9/7.4 years in the 274 patients with a history of AU. The demographic and disease characteristics of all patients and of patients with a history of AU were generally similar. Sulfasalazine treatment was ongoing in 13\% (36/274) of patients with a history of AU and in $10 \%$ (127/1250) of all patients. The percentages of patients with treatment with a previous TNF antagonist was $26 \%(326 / 1250)$ of all patients and $23 \%(63 / 274)$ of patients with a history of AU. The percentage of HLA-B27-positive patients was greater for patients with a history of $A U$ than among the total study population or compared with patients without a history of uveitis, of whom $80 \%$ were HLA-B27 positive (data not shown). The serum concentration of C-reactive protein (CRP) was greater in patients with symptomatic AU at baseline compared with all other patient groups (table 1$)$.

The majority of patients $(84 \%, 231 / 274)$ had experienced acute AU and 16\% (43/274) of patients had developed chronic uveitis. Among the 106 patients with a recent flare of AU, 26 $(25 \%)$ had chronic uveitis. Among the 28 patients with symptomatic uveitis at baseline, 10 (36\%) had a chronic uveitis (table 1).

\section{AU flare rates during the year prior to enrolment}

During the 12 months before and including the baseline visit, 87 patients had experienced 1 to 2 flares of AU and 19 patients had experienced 3 or more episodes of AU. The total number of AU flares in the year before and/or at baseline was 187.5. Accordingly, the AU flare rates per 100 PYs were 15 flares/ 100 PYs in all 1250 patients, 68.4 flares/100 PYs in the 274 patients with a history of uveitis and 176.9 flares/100 PYs in the 106 patients with a recent history of AU. The 28 patients with symptomatic AU at baseline had experienced 54 AU flares in the past year resulting in a rate of 192.9 flares/100 PYs. A total of 55.5 AU flares within the 1 year before adalimumab treatment was documented among the 43 patients diagnosed with chronic uveitis, for an AU flare rate in this group of 129.1 flares/100 PYs (table 2). 
Table 1 Demographic and disease characteristics at baseline of patients with ankylosing spondylitis (AS) and a history of anterior uveitis (AU)

\begin{tabular}{|c|c|c|c|c|c|}
\hline & $\begin{array}{l}\text { All patients } \\
(\mathrm{n}=1250)\end{array}$ & $\begin{array}{l}\text { History of } \mathrm{AU}^{*} \\
(\mathrm{n}=274)\end{array}$ & $\begin{array}{l}\text { Recent history of } \\
\mathrm{AU} \dagger(\mathrm{n}=106)\end{array}$ & $\begin{array}{l}\text { Symptomatic AU at } \\
\text { baseline }(\mathrm{n}=28)\end{array}$ & $\begin{array}{l}\text { Chronic AU: } \\
(\mathrm{n}=43)\end{array}$ \\
\hline Chronic uveitis, \% & 3 & 16 & 25 & 36 & 100 \\
\hline Male, \% & 71 & 70 & 74 & 82 & 72 \\
\hline Age, years, mean (SD) & $43.6(11.4)$ & $45.6(10.6)$ & $44.0(10.0)$ & $46.1(13.0)$ & $44.4(9.9)$ \\
\hline AS duration, years, mean (SD) & $11.0(9.8)$ & $14.8(10.6)$ & $13.4(9.3)$ & $14.8(8.4)$ & $15.1(9.2)$ \\
\hline Advanced AS§, \% & 27 & 31 & 31 & 41 & 26 \\
\hline $\begin{array}{l}\text { Time since onset of uveitis, } \\
\text { years, mean (SD) }\end{array}$ & NA & $9.9(8.9)$ & $6.6(8.8)$ & $7.4(8.4)$ & $9.4(7.9)$ \\
\hline HLA-B27 positive, $\%$ & 82 & 91 & 89 & 82 & 90 \\
\hline BASDAI, mean (SD) & $6.3(1.4)$ & $6.3(1.4)$ & $6.5(1.4)$ & $6.2(1.7)$ & $6.6(1.5)$ \\
\hline BASFI, mean (SD) & $5.4(2.2)$ & $5.2(2.3)$ & $5.0(2.5)$ & $5.0(2.8)$ & $5.2(2.5)$ \\
\hline CRP, mg/dl, mean (SD) & $2.0(2.4)$ & $2.4(2.8)$ & $2.8(3.6)$ & $4.1(4.9)$ & $2.6(3.0)$ \\
\hline Treatment with DMARDs, \% & 26 & 28 & 19 & 32 & 26 \\
\hline $\begin{array}{l}\text { Treatment with } \\
\text { sulfasalazine, \% }\end{array}$ & 12 & 13 & 9 & 25 & 12 \\
\hline Oral NSAIDs, \% & 74 & 79 & 78 & 71 & 84 \\
\hline Oral glucocorticoids, $\%$ & 14 & 13 & 9 & 14 & 16 \\
\hline $\begin{array}{l}\text { Previous etanercept and/or } \\
\text { infliximab treatment, \% }\end{array}$ & 26 & 23 & 26 & 29 & 35 \\
\hline \multicolumn{6}{|c|}{$\begin{array}{l}\text { *History of } \mathrm{AU} \text {, history of at least one } \mathrm{AU} \text { flare anytime. } \\
\text { thecent history of } \mathrm{AU} \text {, history of at least one } \mathrm{AU} \text { flare in the past year. } \\
\text { \#Chronic uveitis refers to persistent AU flares of at least } 3 \text {-month duration and with relapse within less than } 3 \text { months according to } \\
\text { the Standardization of Uveitis Nomenclature Working Group. } \\
\text { \$Advanced AS, radiographically involvement of at least } 50 \% \text { of the spine in }>2 \text { segments. } \\
\text { BASDAl, Bath AS Disease Activity Index; BASFI, Bath AS Functional Index; CRP, C-reactive protein; DMARDs, disease-modifying } \\
\text { antirheumatic drugs; NA, not applicable; NSAIDs, non-steroidal anti-inflammatory drugs. }\end{array}$} \\
\hline
\end{tabular}

\section{Adalimumab exposure}

Among all 1250 patients in this study, the mean/median adalimumab exposure was 106/86 days. Overall, 115 of 1250 $(9.2 \%)$ patients prematurely discontinued from the study. No patient discontinued adalimumab because of an AU episode. The mean/median duration of adalimumab treatment was 129/ 140 days in patients who had at least one AU flare in the 1 year before enrolment and who were observed up to week 20 by protocol.

\section{Reduction of AU flare rates during adalimumab treatment}

During 363 PYs of adalimumab treatment, 27 flares of AU were documented in 25 out of 1250 (2\%) patients. Overall, adalimumab treatment led to clinically relevant reduction in the incidence of AU flares. The rate of uveitis flares dropped by $51 \%$ in all 1250 patients with AS, by $58 \%$ in the 274 patients with a history of $\mathrm{AU}$, by $68 \%$ in those 106 patients with a recent history of $\mathrm{AU}$, by $50 \%$ in the 28 patients with symptomatic AU at baseline and by $45 \%$ in the 43 patients with a history of chronic uveitis (table 2).

Compared with the control period, the incidence of AU flares in the complete study population was decreased for $7.3 \%$ (91/ $1250)$ of patients, unchanged for $91.0 \%$ (1138/1250) of patients and increased for $1.7 \%(21 / 1250)$ of patients.

A total of 25 AU flares were documented in 23 of 274 patients with a history of AU; 21 AU flares occurred in 19 of 106 patients with a recent history of $\mathrm{AU}$. Of the 19 patients who had reported at least 3 AU flares in the past year, 11 (58\%) had no relapse of AU during adalimumab treatment. Of 87 patients who had experienced 1 to 2 AU flares in the past year, $76(87 \%)$ had no AU flares during adalimumab treatment. Among the 28 patients with symptomatic AU at baseline, 19 (68\%) experienced no AU flares during adalimumab treatment; 10 AU flares were reported in the other 9 patients.

Table 2 Anterior uveitis flare rates per 100 patient years before and during adalimumab therapy

\begin{tabular}{|c|c|c|c|c|}
\hline $\begin{array}{l}\text { Patient groups by } \\
\text { history of } \mathrm{AU}\end{array}$ & $\begin{array}{l}\text { AU flare rate before } \\
\text { adalimumab treatment } \\
\text { (flares/100 PYs) }\end{array}$ & $\begin{array}{l}\text { AU flare rate during } \\
\text { adalimumab treatment } \\
\text { (flares/100 PYs) }\end{array}$ & $\begin{array}{l}\text { Reduction in AU flare } \\
\text { rate during } \\
\text { adalimumab treatment } \\
(\%)\end{array}$ & p Value* \\
\hline $\begin{array}{l}\text { All patients } \\
(\mathrm{n}=1250)\end{array}$ & 15 & 7.4 & -51 & $<0.001$ \\
\hline $\begin{array}{l}\text { History of } \mathrm{AU} \dagger \\
(\mathrm{n}=274)\end{array}$ & 68.4 & 28.9 & -58 & $<0.001$ \\
\hline $\begin{array}{l}\text { Recent history of } \\
\operatorname{AU} \$(n=106)\end{array}$ & 176.9 & 56 & -68 & $<0.001$ \\
\hline $\begin{array}{l}\text { Symptomatic AU at } \\
\text { baseline }(n=28)\end{array}$ & 192.9 & 96.2 & -50 & 0.001 \\
\hline $\begin{array}{l}\text { Previous chronic } \\
\text { uveitis } \S(n=43)\end{array}$ & 129.1 & 71.4 & -45 & 0.002 \\
\hline
\end{tabular}

*Wilcoxon signed rank test.

+History of $\mathrm{AU}$, history of at least one $\mathrm{AU}$ flare anytime. + Recent history of $\mathrm{AU}$, history at least one $\mathrm{AU}$ flare in the past year. $\S$ Chronic uveitis refers to persistent AU flares of at least 3-month duration and with relapse within less than 3 months according to the Standardization of Uveitis Nomenclature Working Group. ${ }^{6}$

$\mathrm{AU}$, anterior uveitis; PYs, patient years. 
In general, the percentage of patients who experienced an AU flare during adalimumab treatment was greater in patients with a history of chronic uveitis $(21 \%, 9 / 43)$ than in patients with a history of acute $\mathrm{AU}(6 \%, 14 / 231)$. Each of the nine patients with chronic uveitis and with an AU flare during adalimumab treatment had experienced uveitis flares within the year before study enrolment.

Among all patients $(n=1250), 2$ developed new-onset AU. In one patient, a mild $\mathrm{AU}$ flare occurred 4 days after the first adalimumab injection and ceased after 11 days topical nonsteroidal treatment. Simultaneously with the remission of uveitis, the patient responded well to adalimumab, as indicated by a decrease in BASDAI from 6.3 at baseline to 1.1 at week 2 . In the other patient, a moderate flare of AU occurred 90 days after initiation of adalimumab therapy. The AU flare in this patient was associated with a deterioration of AS activity, as suggested by an increase in BASDAI from 2.1 at week 6 to 4.7 at week 12 and by an increase of CRP concentration from $0.2 \mathrm{mg} / \mathrm{dl}$ at week 6 to $1.2 \mathrm{mg} / \mathrm{dl}$ at week 12 .

The majority of AU flares that occurred during adalimumab treatment were reported as mild (67\%) or moderate (29\%); only one flare was considered severe by the treating doctor. All patients recovered from $\mathrm{AU}$ after topical anti-inflammatory treatment except for two patients with ongoing $\mathrm{AU}$ at the last observation. In one of these two patients, the AU flare had started 1 week after the last adalimumab injection; in the other patient, the onset of the AU flare was reported 2 months before the last observation. The time from first injection of adalimumab until onset of AU flares varied between 4 and 113 days, with a median interval of 72 days. The median duration of the 25 AU flare episodes that had a documented date of resolution was 22 days.

\section{ASAS and BASDAI responses}

The response of AS signs and symptoms to adalimumab was similar in all patients and in patients with a history of AU (table 3 ).

At week 12, 46\% of the 25 patients who had an AU flare during adalimumab treatment achieved an ASAS40 response compared with $54 \%$ of the 1225 patients who had no AU flare during adalimumab treatment. The BASDAI 50 response rates were $56 \%$ of 25 patients who had an AU flare during adalimumab treatment and $57 \%$ of 1225 patients who had no AU flare during adalimumab treatment. Of the 25 patients who experienced an AU flare during adalimumab treatment, 7 (28\%) were either insufficient responders with respect to their AS disease during the entire adalimumab treatment period, as indicated by a change from baseline in BASDAI $<20 \%$, or had a relapse of AS activity before the AU flare, indicated by at least a $50 \%$ increase in the BASDAI compared with the BASDAI score at the preceding study visit (data not shown). In three patients, the AU flare occurred during the 70-day follow-up period; therefore, no information on AS disease activity was available.

\section{DISCUSSION}

Our results suggest at least a 50\% reduction in the AU flare rate during adalimumab treatment of patients with active AS. The results of this study are somewhat limited by the open-label, uncontrolled study design but are strengthened by the large number $(>1000)$ of patients cared for in 211 centres in 15 European countries. Of the 1250 enrolled patients with AS, 274 had a history of AU. The AU flare rate of $15 / 100$ PYs in all 1250 patients with AS before adalimumab treatment is similar to the AU flare rate of 15.6/100 PYs reported by Braun and colleagues in placebo-treated patients with AS from three pooled clinical trials of etanercept or infliximab for AS (the range among the three placebo groups varied from 0 to $37.2 / 100$ PYs). ${ }^{17}$ Treatment with adalimumab decreased the rate of AU flares by $51 \%$ for all 1250 patients with AS to an AU flare rate of 7.4/ 100 PYs. By comparison, Braun and colleagues ${ }^{17}$ reported a pooled AU flare rate of 3.4/100 PYs for all infliximab-treated patients (range from 2.2 to $18.9 / 100$ PYs in the three infliximab studies) and a pooled AU flare rate of 7.9/100 PYs for etanercept-treated patients (range from 0 to $9.6 / 100$ PYs in four etanercept studies). Information about the AU flare rates before enrolment in these clinical studies of etanercept and infliximab was not collected.

For the 274 patients in this study with a history of AU, the rate of AU flares decreased by $58 \%$, from 68.4/100 PYs to $28.9 /$ 100 PYs during adalimumab treatment. This rate is comparable to the rate of $21.4 / 100$ PYs in a retrospective study of 46 TNF antagonist-treated patients with AS or with SpA. ${ }^{18}$ The majority of AU flares during the study were reported for those 106 patients who had a recent history of AU (ie, at least 1 flare within the past year). Nevertheless, in this subset of patients at high risk for relapse of $\mathrm{AU}$, the rate of $\mathrm{AU}$ flares was reduced by $68 \%$ during adalimumab therapy. Also, in patients with a history of chronic uveitis, a reduction of AU flares during the study was observed. Most of the AU flares that occurred during adalimumab treatment were considered mild and subsided after 3 weeks in half of the patients in whom the duration of the AU flare was known.

Table 3 Adalimumab effectiveness at week 12 in all patients and in patients with a history of anterior uveitis (observed values)

\begin{tabular}{|c|c|c|c|c|c|}
\hline & $\begin{array}{l}\text { All patients } \\
(n=1250)\end{array}$ & $\begin{array}{l}\text { History of } \mathrm{AU}^{*} \\
(\mathrm{n}=274)\end{array}$ & $\begin{array}{l}\text { Recent history of } \\
\mathrm{AU} \dagger(\mathrm{n}=106)\end{array}$ & $\begin{array}{l}\text { Symptomatic AU at } \\
\text { baseline }(n=28)\end{array}$ & $\begin{array}{l}\text { Chronic } A U: \\
(n=43)\end{array}$ \\
\hline ASAS40, \% & 53.7 & 54.5 & 55.1 & 56.0 & 52.4 \\
\hline BASDAI 50, \% & 57.2 & 60.7 & 61.8 & 66.7 & 59.5 \\
\hline $\begin{array}{l}\text { ASAS partial } \\
\text { remission, } \%\end{array}$ & 27.7 & 32.5 & 32.7 & 40.0 & 33.3 \\
\hline $\begin{array}{l}\text { CRP, mg/dl, mean } \\
\text { (SD) }\end{array}$ & $0.8(1.4)$ & $1.0(1.8)$ & $1.2(2.2)$ & $1.6(2.6)$ & $0.9(0.9)$ \\
\hline $\begin{array}{l}\text { CRP absolute change, } \\
\text { mean (SD) }\end{array}$ & $-1.4(2.5)$ & $-1.9(3.3)$ & $-2.3(4.5)$ & $-3.8(5.5)$ & $-2.6(3.2)$ \\
\hline
\end{tabular}

*History of $\mathrm{AU}$, history of at least one AU flare anytime.

$\uparrow$ Recent history of $A U$, history of at least one AU flare in the past year. $\$$ Chronic uveitis refers to persistent AU flares of at least 3 month duration and with relapse within less than 3 months according to the Standardization of Uveitis Nomenclature Working Group. ${ }^{6}$

ASAS, Assessments in Spondyloarthritis International Society criteria for response, partial remission (value of $<2$ on a 0-10-point scale in each of the four ASAS20 domains); AU, anterior uveitis; BASDAI, Bath Ankylosing Spondylitis Disease Activity Index; CRP, C-reactive protein. 
The general therapeutic response of AS signs and symptoms in patients with a history of $\mathrm{AU}$ was comparable to the response of all patients in the study. The comparison of adalimumab effectiveness in patients with or without an AU flare during treatment is limited because of the large difference in the number of patients in the 2 groups ( 25 who had an AU flare vs 1225 who did not) and because of the uncontrolled study design. However, an insufficient response or loss of response to adalimumab as measured by AS disease activity was documented in more than $25 \%$ of patients at the time of the AU flares. Nevertheless, most AU flares occurred in patients with a good overall clinical response to adalimumab, suggesting that vigilant monitoring for AU flares is necessary in patients with AS whose disease activity is low.

Two male patients developed an episode of new-onset unilateral AU. New-onset AU has been reported in adult and paediatric patients treated with etanercept ${ }^{19-21}{ }^{33}$ and rarely in patients treated with infliximab. ${ }^{23} 333^{34}$ The underlying rheumatic disorders in patients with new-onset AU were often diseases that are not commonly associated with $\mathrm{AU}$, such as rheumatoid arthritis. ${ }^{24}$ Atypical bilateral AU flares were reported in six patients with AS during anti-TNF therapy (five patients with etanercept treatment, one patient with infliximab treatment). ${ }^{23}{ }^{35}$ Because $\mathrm{AU}$ is frequently comorbid with $\mathrm{AS}$, it is difficult to determine whether the underlying AS or the antiTNF treatment has caused the new-onset AU. In one of the patients in the study, the first episode of AU occurred 4 days after the first adalimumab injection. Although a temporal relationship between adalimumab injection and the AU flare is apparent, this patient was in a state of high AS disease activity at the time of the flare. The same consideration is likely for the second patient who developed an AS flare 5 days before the first AU flare. On the other hand, the correlation between AS disease activity and the incidence of AU flares is generally considered to be low. ${ }^{2}$

During adalimumab treatment, the rate of AU flares was reduced by $51 \%$ in all patients, by $58 \%$ in patients with a history of $\mathrm{AU}$, by $68 \%$ in patients with a recent history of $\mathrm{AU}$, by $50 \%$ in patients with symptomatic AU at baseline and by $45 \%$ in patients with chronic uveitis. Most AU flares that occurred during adalimumab treatment were mild, and only two patients had new-onset AU.

\section{CONCLUSIONS}

Adalimumab effectively reduces the rate of AU flares in patients with active AS, including patients with recently symptomatic $\mathrm{AU}$ and patients with chronic uveitis.

Acknowledgements: We thank all principal investigators and research nurses who
contributed to patient recruitment and data collection in RHAPSODY. We are grateful
to the following for enrolling at least three patients with anterior uveitis. Austria:
Professor Dr M Herold (Innsbruck); Belgium: Dr J Vanhoof (Genk); Denmark: Dr K Grau
(Kolding); Dr P Holck (Silkeborg); France: Dr C Behamou (Orleans); Dr T Billey (Cahors);
Dr D Briancon (Aix-les-Bains); Professor Dr P Claudepierre (Creteil); Professor Dr P
Fardellone (Amiens); Dr J Godde (Marseille); Dr J-L Grauer (Aix-en-Prevence);
Professor Dr A Saraux (Brest); Germany: Professor Dr J Braun (Herne); Professor Dr G
Hein (Jena); Professor Dr H Kellner (München); Professor Dr A Krause (Berlin); Dr K
Rockwitz (Goslar); Professor Dr A Rubberth-Roth (Köln); Dr W Spieler (Zerbst); Dr Dr H
Tremel (Hamburg); Greece: Professor Dr D Boumpas (Herakleion); Norway: Dr E
Rødevand (Trondheim); Spain: Dr A Alonso (Barakaldo); Dr P Fernandez (Madrid); Dr E
Loza (Pamplona); Dr C Rodriguez (Las Palmas de Gran Canaria); Switzerland: Dr R
Theiler (Zürich); UK: Dr E Choy (London); Dr R Cooper (Manchester); Dr C Edwards
(Southampton); Professor Dr P Emery (Leeds); Dr A Keat (Harrow); Professor Dr R
Moots (Liverpool). We also thank loanna Mantika for study management (Abbott, UK);
Christa Zaiti-Runkel for data management; Angelika Freitag and Anja Bruhn for
statistical programming (both Abbott GmbH \& Co. KG, Germany); and Dana L Randall
for editing the manuscript (JK Associates, Inc., Conshohocken, Philadelphia, USA).
Funding: The research reported here and the preparation of this manuscript were funded by Abbott Laboratories. The RHAPSODY Study Group included experts from academic institutions in Europe and the USA and members of Abbott Laboratories who designed the original clinical trial. Clinical data were collected and analysed by Abbott Laboratories. All authors contributed to manuscript development and reviewed and approved the content of the submitted manuscript.

Competing interests: MR, ER, PH and JV were RHAPSODY study investigators. MK and $\mathrm{HK}$ are employees of Abbott $\mathrm{GmbH} \& \mathrm{Co} \mathrm{KG}$, an affiliate of Abbott Laboratories. They hold shares of Abbott stock. SK is a contractor of Abbott GmbH.

Ethics approval: This study was approved by an independent ethics committee at each centre and all patients provided written, informed consent.

\section{REFERENCES}

1. Rosenbaum JT, Smith JR. Anti-TNF therapy for eye involvement in spondyloarthropathy. Clin Exp Rheumatol 2002;20(Suppl 28):S143-5.

2. Hamideh F, Prete PE. Ophthalmologic manifestations of rheumatic diseases. Semin Arthritis Rheum 2001;30:217-41.

3. Pato E, Banares J, Jover JA, Fernandez-Gutierrez B, Godoy F, Morado C, et al. Undiagnosed spondyloarthropathy in patients presenting with anterior uveitis. J Rheumatol 2000;27:2198-202

4. Linder R, Hoffmann A, Brunner R. Prevalence of the spondylarthritides in patients with uveitis. J Rheumatol 2004;31:2226-9.

5. Monnet D, Breban M, Hudry C, Dougados M, Brézin AP. Ophthalmic findings and frequency of extraocular manifestations in patients with HLA-B27 uveitis: a study of 175 cases. Ophthalmology 2004;111:802-9.

6. Jabs DA, Nussenblatt RB, Rosenbaum JT. Standardization of Uveitis Nomenclature (SUN) Working Group. Standardization of uveitis nomenclature for reporting clinical data. Results of the First International Workshop. Am J Ophthalmol 2005;140:509-16.

7. Benitez del Castillo JM, Garcia Sanchez J, Tradier T, Banares A. Sulfasalazine in the prevention of anterior uveitis associated with ankylosing spondylitis. Eye 2000;14:340-3.

8. El-Shabrawi Y, Hermann J. Anti-tumor necrosis factor- $\alpha$ therapy with infliximab as an alternative to corticosteroids in the treatment of human leukocyte antigen B27associated acute anterior uveitis. Ophthalmology 2002;109:2342-6.

9. Smith JR, Levinson RD, Holland GN, Jabs DA, Robinson MR, Whitcup SM, et al. Differential efficacy of tumor necrosis factor inhibition in the management of inflammatory eye disease and associated rheumatic disease. Arthritis Rheum 2001;45:252-7.

10. Smith JA, Thompson DJS, Whitcup SM, Suhler E, Clarke G, Smith S, et al. A randomized, placebo-controlled, double-masked clinical trial of etanercept for the treatment of uveitis associated with juvenile idiopathic arthritis. Arthritis Rheum 2005; 53:18-23

11. Saurenmann RK, Levin AV, Rose JB, Parker S, Rabinovitch T, Tyrrell PN, et al. Tumour necrosis factor $\alpha$ inhibitors in the treatment of childhood uveitis. Rheumatology Oxford 2006;45:982-9.

12. Hale S, Lightman S. Anti-TNF therapies in the management of acute and chronic uveitis. Cytokine 2006;33:231-7.

13. Kahn P, Weiss M, Imundo LF, Levy DM. Favorable response to high-dose infliximab for refractory childhood uveitis. Ophthalmology 2006;113:860-4.

14. Sobrin L, Kim EC, Christen W, Papadaki T, Letko E, Foster CS. Infliximab therapy for the treatment of refractory ocular inflammatory disease. Arch Ophthalmol 2007; 125:895-900.

15. Tynhälä $\mathbf{P}$, Lindahl $P$, Honkanen V, Lahdenne $P$, Kotaniemi K. Infliximab and etanercept in the treatment of chronic uveitis associated with refractory juvenile idiopathic arthritis. Ann Rheum Dis 2007;66:548-50.

16. Galor A, Perez VL, Hammel JP, Lowder CY. Differential effectiveness of etanercept and infliximab in the treatment of ocular inflammation. Ophthalmology 2006;116:2317-23.

17. Braun J, Baraliakos X, Listing J, Sieper J. Decreased incidence of anterior uveitis in patients with ankylosing spondylitis treated with the anti-tumor necrosis factor agents infliximab and etanercept. Arthritis Rheum 2005;52:2447-51.

18. Guignard S, Gossec L, Salliot C, Ruyssen-Witrand A, Luc M, Duclos M, et al. Efficacy of tumour necrosis factors blockers in reducing uveitis flares in spondyloarthropathy: a retrospective study. Ann Rheum Dis 2006;65:1631-4.

19. Tiliakos AN, Tiliakos NA. Ocular inflammatory disease in patients with RA taking etanercept: is discontinuation of etanercept necessary? J Rheumatol 2003;30:2727.

20. Hashkes PJ, Shajrawi I. Sarcoid-related uveitis occurring during etanercept therapy. Clin Exp Rheumatol 2003:21:645-6.

21. Reddy AR, Backhouse OC. Does etanercept induce uveitis? Br J Ophthalmol 2003; 87:925.

22. Foster CS, Tufail F, Waheed NK, Chu D, Miserocchi E, Baltatzis S, et al. Efficacy of etanercept in preventing relapse of uveitis controlled by methotrexate. Arch Ophthalmol 2003;121:437-40.

23. Monnet D, Moachon L, Dougados M, Brézin AP. Severe uveitis in an HLA-B27positive patient with ankylosing spondylitis. Nat Clin Pract Rheumatol 2006;2:393-7.

24. Lim LL, Fraunfelder FW, Rosenbaum JT. Do tumor necrosis factor inhibitors cause uveitis? A registry-based study. Arthritis Rheum 2007;56:3248-52.

25. Van der Linden S, Valkenburg HA, Cats A. Evaluation of diagnostic criteria for ankylosing spondylitis: a proposal for modification of the New York criteria. Arthritis Rheum 1984;27:361-8. 
26. Garrett S, Jenkinson T, Kennedy LG, Whitelock H, Gaisford P, Calin A. A new approach to defining disease status in ankylosing spondylitis: the Bath Ankylosing Spondylitis Disease Activity Index. J Rheumatol 1994;21:2286-91.

27. Rudwaleit M, Claudepierre P, Wordsworth P, Loza E, Wong R, Kron M, et al. Predictors of major clinical responses (BASDAI50, ASAS40, ASAS partial remission) in 1,250 patients treated with adalimumab (HUMIRA) for active ankylosing spondylitis. Ann Rheum Dis 2008;67(Suppl 2):516.

28. Braun J, van der Heijde D, Dougados M, Emery P, Khan MA, Sieper J, et al. Staging of patients with ankylosing spondylitis: a preliminary proposal. Ann Rheum Dis 2002;61(Suppl 3):19-23.

29. Anderson JJ, Baron G, van der Heijde D, Felson DT, Dougados M. Ankylosing spondylitis assessment group preliminary definition of short-term improvement in ankylosing spondylitis. Arthritis Rheum 2001;44:1876-86.

30. Brandt J, Listing J, Sieper J, Rudwaleit M, van der Heijde D, Braun J. Development and preselection of criteria for short term improvement after anti-TNF $\alpha$ treatment. Ann Rheum Dis 2004;63:1438-44.
31. Braun J, Pham T, Sieper J, Davis J, van der Linden S, Dougados M, et al. International ASAS consensus statement for the use of anti-tumour necrosis factor agents in patients with ankylosing spondylitis. Ann Rheum Dis 2003;62:817-24.

32. Braun J, Davis J, Dougados M, Sieper J, van der Linden S, van der Heïde D, ASAS Working Group. First update of the international ASAS consensus statement for the use of anti-TNF agents in patients with ankylosing spondylitis. Ann Rheum Dis 2006:65:316-20.

33. Di Gangi M, Foti R, Leonardi R, Leonetti C, Castellino P. Recurrent new-onset uveitis in a patient with rheumatoid arthritis during anti-TNF $\alpha$ treatment. Reumatismo 2007:59:169-72.

34. Kaipiainen-Seppänen 0, Leino M. Recurrent uveitis in a patient with juvenile spondyloarthropathy associated with tumour necrosis factor $\alpha$ inhibitors. Ann Rheum Dis 2003;62:88-9.

35. Coates LC, McGonagle DG, Bennett AN, Emery P, Marzo-Ortega. Uveitis and tumour necrosis factor blockade in ankylosing spondylitis. Ann Rheum Dis 2008;67:729-30. 